\title{
Compartmentalization of Gold Nanocrystals in Polymer Microparticles using Electrohydrodynamic Co-Jetting ${ }^{\mathrm{a}}$
}

\author{
Dong Woo Lim, Sangyeul Hwang, Oktay Uzun, Francesco Stellacci, \\ Joerg Lahann*
}

Polymer particles with micro- and nanoscale anisotropy have received increasing interest for their ability to simultaneously present different physical- and chemical properties. In this communication, we demonstrate that gold nanocrystals (NCs) can be selectively incorporated into one compartment of anisotropic polymer particles. Stable bicompartmental particles were prepared via electrohydrodynamic co-jetting of aqueous nanoparticle suspensions followed by thermal cross-linking. Bicompartmental particle populations with different NC densities were obtained by varying the NC concentration in the jetting suspension. While NCloaded polymer particles showed different optical properties depending on the NC density, they still maintained discrete interfaces between two compartments. Moreover, the fraction of the bicompartmental particles was higher than $98 \%$ based on flow cytometry. This study delineates a new approach for preparation of inorganic/organic composite particles with precisely engineered, anisotropic nanoparticle distributions and may contribute to further developments in emerging scientific areas, such as smart materials or particle-based diagnostics.

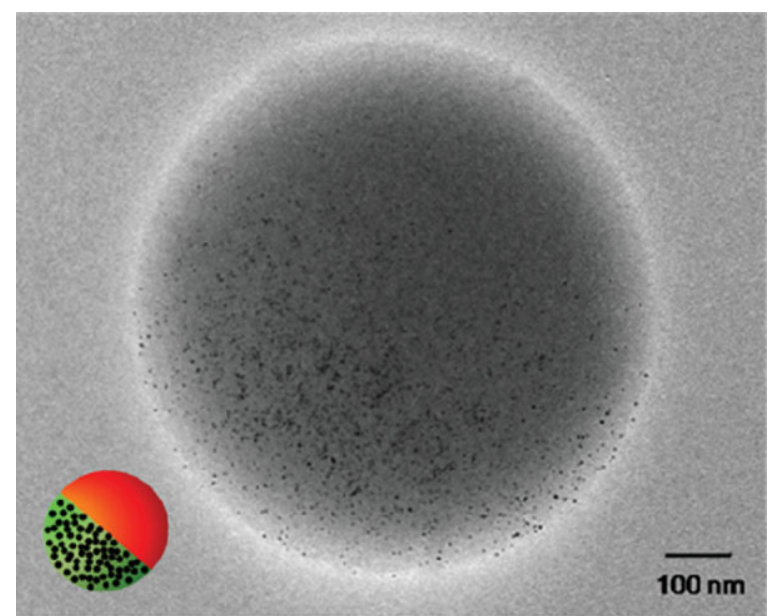

D. W. Lim, S. Hwang, J. Lahann

Departments of Chemical Engineering, Macromolecular Science and Engineering, and Materials Science and Engineering,

University of Michigan, Ann Arbor, Michigan 48109, USA

Fax: (+1) 734764 7453; E-mail: lahann@umich.edu

O. Uzun, F. Stellacci

Department of Materials Science and Engineering,

Massachusetts Institute of Technology, Massachusetts 02139,

USA

${ }^{\mathrm{a}}$ Supporting information for this article is available at the bottom of the article's abstract page, which can be accessed from the journal's homepage at http://www.mrc-journal.de, or from the author.

\section{Introduction}

Design and synthesis of anisotropic polymer particles with micro- and nanoscale compartments have been burgeoning areas of contemporary materials research. ${ }^{[-3]}$ Ultimately the controlled introduction of multiple functional nanomaterials gives rise to different physical and chemical properties, and may be of potential importance for the development of smart polymeric materials in industrial and biomedical applications. ${ }^{[2-4]}$ Specifically, bicompartmental particles with different optical, electric, and magnetic characteristics within different compartments 
have been pursued for their potential to undergo switching in response to electrical or magnetic fields, as well as light-, temperature-, or pH-based changes..$^{[3,5-9]}$

So far, most of the interest related to anisotropic particles has been focused on the synthesis of Janus particles and a number of methods have been reported for the preparation of Janus particles including microfluidic techniques, flowfocusing lithography, the use of spinning disks, selective deposition, modification by partial masking, pickering emulsion, and self-assembly. ${ }^{[10-27]}$ Recently, electrohydrodynamic (EHD) co-jetting of multiple polymer solutions with a side-by-side geometry has been used for synthesis of anisotropic particles and fibers with two or more distinct bulk compartments and surfaces. ${ }^{[11,12,28,29]}$ Compared to above-mentioned processes, EHD co-jetting offers a number of potential advantages, specifically variable multiplexing of particle attributes and scale-up for fabrication of micronor submicron-sized anisotropic particles. ${ }^{[30]}$ We recently reported that anisotropic nano- and microparticles, fibers, and cylinders with bi-, tri-, and multicompartmental architectures can be prepared by EHD co-jetting using an appropriate number of parallel liquid flows. ${ }^{[11,12,28]}$ Similarly, environmentally responsive core-shell particles were prepared using fully miscible polymeric solutions. ${ }^{[31]} \mathrm{We}$ also demonstrated that distinct surface areas of chemically anisotropic particles and fibers can be selectively addressed by spatio-selective immobilization of streptavidin or Huisgen 1,3-dipolar cycloaddition. ${ }^{[10,32,33]}$ Herein, we show that functional nanomaterials, i.e., gold nanocrystals (NCs) with an average diameter of $4 \mathrm{~nm}$, can be selectively compartmentalized into one hemisphere of anisotropic polymer particles.

\section{Experimental Part}

\section{Electrohydrodynamic Co-Jetting with a Side-by-Side Geometry}

Two polymer solutions were separately prepared in double distilled, deionized water: (i) $7.0 \mathrm{w} / \mathrm{v} \%$ poly(acrylamide-co-acrylic acid, sodium salts), P(AAm-co-AA) (MW $200 \mathrm{kDa} ; 10 \%$ acrylic acids; Polysciences, Warrington, PA, US), and $0.5 \mathrm{w} / \mathrm{v} \%$ rhodamine $\mathrm{B}$ isothiocyanate conjugated dextran, RITC-dextran (MW $70 \mathrm{kDa}$; Sigma-Aldrich, US) in water; (ii) $7.0 \mathrm{w} / \mathrm{v} \% \mathrm{P}(\mathrm{AAm}-\mathrm{CO}-\mathrm{AA})$, and $0.5 \mathrm{w} / \mathrm{v} \%$ fluorescein isothiocyanate (FITC) conjugated dextran, FITC-dextran (MW $70 \mathrm{kDa}$; Sigma-Aldrich, US) with various final concentrations of the gold NCs $(0,0.18,0.36$, and $0.72 \mathrm{w} / \mathrm{v} \%)$ in water. The gold NCs capped with 11-mercapto-1-undecanesulfonate (MUS) and functionalized with 11-mercaptoundecanoic acids (MUAs) at polar defects were synthesized, as published previously. ${ }^{[34-37]}$ Conductivity of the final polymer solutions was measured by the Orion 105 Plus Conductivity Meter (Thermo Electron, US). Figure 1(A) shows a schematic of an experimental setup of the EHD co-jetting with a side-by-side geometry of dual capillaries for synthesis of anisotropic particles. The polymer jetting solutions were separately loaded in $1 \mathrm{ml}$ syringes (BectonDickinson, New Jersey, US) and two syringes were connected to a dual channel tip (FibriJet SA-3610, Micromedics, MN, US) having two capillaries with 26 gauge and 3.25 inch in length. A dual syringe applicator assembly (FibriJet SA-0100, Micromedics, MN, US) was used to move two syringes at the same time. The cathode of the high-voltage supply (Gamma High Voltage Research, US) was attached to the two capillaries together, while the counterelectrode was connected to the collecting substrate of an aluminum (Al) foil (Fisherbrand, US). The distance between the tip of the capillaries and the collecting substrate was vertically maintained in the range of $28-30 \mathrm{~cm}$. The DC electrical potential was applied in the range of 15-18 kV during the EHD co-jetting. All experiments of the EHD cojetting were performed under ambient conditions.

\section{Characterization of Bicompartmental Particles}

Bicompartmental polymer particles situated on the collecting substrate were incubated at $175^{\circ} \mathrm{C}$ overnight to ensure thermal cross-linking of the $\mathrm{P}(\mathrm{AAm}-\mathrm{Co}-\mathrm{AA})$ polymer chains. ${ }^{[13,14]}$ Thermally cross-linked particles were harvested from the collecting substrate,
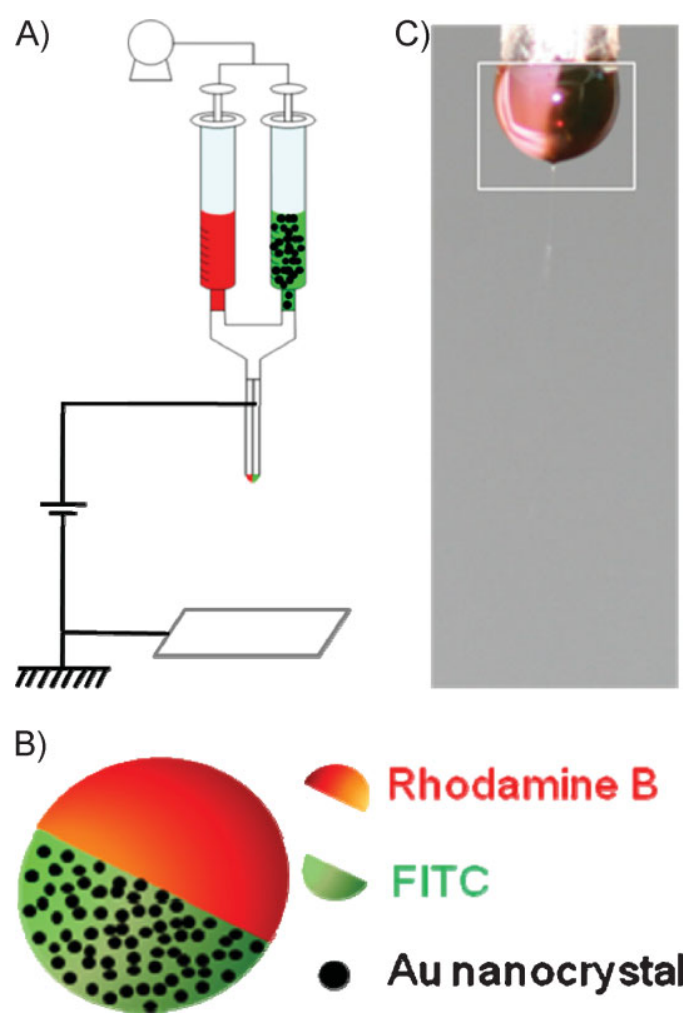

Figure 1. (A) A schematic of EHD co-jetting by side-by-side dual capillaries for synthesis of bicompartmental polymer particles with gold NCs confined in one compartment, (B) cartoon of an anisotropic particle, in which the gold NCs are localized in one hemisphere, and two dyes are compartmentalized into respective hemispheres, and (C) a photographic image of a Taylor cone showing an interface between two polymer solutions and its liquid jet during EHD co-jetting. 
suspended in water or phosphate-buffered saline (PBS), and sonicated for 30-60 s prior to characterization. Confocal laser scanning microscopy (CLSM, Olympus FluoView 500, US) was used to analyze the degree of anisotropy of the particles having two different fluorescent dyes (FITC-dextran and RITC-dextran). An Ar/ArKr laser at $488 \mathrm{~nm}$ and a GreNe laser at $543 \mathrm{~nm}$ were used to excite FITC and rhodamine B respectively, and the emission wavelength ranges were adjusted to $508-523 \mathrm{~nm}$ for FITC and 580$595 \mathrm{~nm}$ for rhodamine B. In addition, differential interference contrast (DIC) imaging was performed to characterize swollen particles in PBS at pH 7.4. Moreover, scanning electron microscopy (SEM, FEI Nova Nanolabs) and transmission electron microscopy (TEM, JEOL 3011) were used to characterize the particles with respect to size and size distributions as a function of different densities of the gold NCs between two compartments within the same particles. Various aqueous particle suspensions were centrifuged and the precipitates were suspended in ethanol, and dried on Al foil for SEM images or a copper TEM grid coated with a carbon film (400 meshes, Ted Pella, US) for TEM images. SEM images were obtained at $10 \mathrm{kV}$ without any gold deposition for introducing a conductive layer, and TEM images were obtained with an accelerating voltage of $300 \mathrm{kV}$. Average diameters of the particles were determined by measuring approximately $400-500$ particles in randomly selected areas of the SEM images. Finally, the anisotropic particles having different NC densities in one compartment were suspended in water under the identical concentration of $0.67 \mathrm{mg} \cdot \mathrm{ml}^{-1}$ and their optical images were taken by a digital camera.

\section{Flow Cytometry Analysis of Bicompartmental Particles}

Two separate batches of bicompartmental particles were prepared using $\mathrm{P}(\mathrm{AAm}-\mathrm{Co}-\mathrm{AA})$ as mentioned above. The gold NCs were suspended at the concentration of $0.72 \mathrm{w} / \mathrm{v} \%$ in the one aqueous solution for both batches. The first sample contained FITC-dextran and Atto 647-conjugated dextran at respective compartments as well as gold NCs loaded into the FITC-dextran compartment. The second sample contained the gold NCs at one compartment, but no dyes. Atto 647 (Fluka, Germany) was conjugated with aminodextran (MW $70 \mathrm{kDa}$, Molecular Probes, US) by using 1-ethyl-3-[3dimethylaminopropyl]carbodiimide (EDC, Pierce, US) and sulfo- $N$-hydroxysulfosuccinimide (sulfo-NHS Pierce, US). Atto 647-conjugated dextran was purified by dialysis (MW cutoff of a membrane, $3400 \mathrm{Da}$; Fisherbrand, US) for $3 \mathrm{~d}$ and then freeze-dried. The bicompartmental polymer particles were suspended in PBS at the particle concentration of $0.33 \mathrm{mg} \cdot \mathrm{ml}^{-1}$, and were analyzed by a FACS Vantage SE (BD Biosciences, San Jose, CA) at the Flow Cytometry Core Facility of the University of Michigan. The individual signals of the FITC and Atto 647 were resolved in FL1 channel (excitation by $488 \mathrm{~nm}$ with an Ar laser and emission with $530 / 30 \mathrm{~nm}$ bandpass filter) and FL4 channel (excitation by $633 \mathrm{~nm}$ with a He/Ne laser and emission with $661 / 16 \mathrm{~nm}$ bandpass filter), respectively. Data acquisition and analysis with 20000 events per each sample were performed using CellQuest Pro (BD Biosciences) and WinMDI (Scripps Research Institute, La Jolla, CA). Density plots of FL1 versus FL4 signal intensities were represented for quantitative analysis of the bicompartmental particles. Reproducibility of the density plots was examined by three replicate samples.

\section{Results and Discussion}

Figure 1(A) illustrates a schematic of the experimental setup for EHD co-jetting with a side-by-side geometry featuring two capillaries. This set-up was used for synthesis of anisotropic polymer particles. Two aqueous solutions for EHD co-jetting were individually prepared and loaded into two parallel syringes. One solution contained FITC-dextran, $\mathrm{P}(\mathrm{AAm}-\mathrm{CO}-\mathrm{AA})$, and gold NCs capped with MUS, while the second solution contained RITC-dextran and P(AAm-co-AA), but no gold NCs. The two fluorescent dyes are used to analyze the degree of anisotropy of the resultant polymer particles after thermal cross-linking by CLSM. Figure 1(B) depicts an anisotropic particle, in which the gold NCs are well distributed with very little aggregation in one hemisphere, and both fluorescence dyes are compartmentalized into respective hemispheres. After EHD co-jetting, the organic/inorganic composite particles are stabilized by thermally cross-linking $\mathrm{P}(\mathrm{AAm}-\mathrm{CO}-\mathrm{AA})$ at $175^{\circ} \mathrm{C}^{[13]}$

As shown in Figure 1(C), a stable Taylor cone was formed and the interface between two polymer solutions was maintained under DC electrical potential during the preparation of the bicompartmental polymer particles. ${ }^{[1]}$ In accordance with previous reports, the swirling motion in the Taylor cone was minimized at high viscosity and conductivity of the jetting solutions. ${ }^{[38-40]}$ Specifically, a discrete interface between two polymer solutions in the Taylor cone was maintained at a DC potential in the range of $15-18 \mathrm{kV}$. Meanwhile, conductivities of the two polymer solutions were selected to be in the range of 3.5$3.8 \mathrm{mS} \cdot \mathrm{cm}^{-1}$, irrespective of the presence of the gold NCs in only one jetting solution. Due to optimized viscosity and balanced conductivity of the jetting solutions, bicompartmental particles were prepared, in which the gold NCs were homogeneously distributed in one compartment without any severe aggregation; even after thermal cross-linking at $175^{\circ} \mathrm{C}$ (see below and Figure 2).

To assess a range of gold NC concentration that can be selectively loaded within one of the compartments, a series of experiments were performed with varying concentration of the gold NCs (Control: $0 \mathrm{w} / \mathrm{v} \%, 1: 0.18 \mathrm{w} / \mathrm{v} \%$, $2: 0.36 \mathrm{w} / \mathrm{v} \%$, and $3: 0.72 \mathrm{w} / \mathrm{v} \%$ in one jetting solution), while keeping other parameters constant. As a result, Figure 2(A) represents photographic images of a series of the thermally cross-linked particles suspended in water. The suspensions with a particle concentration of $0.67 \mathrm{mg} \cdot \mathrm{ml}^{-1}$ exhibited significant color differences, implicating that the elevated intensity of pink color in the particle suspension was caused by the increased densities of the gold NCs 
A) Control 1

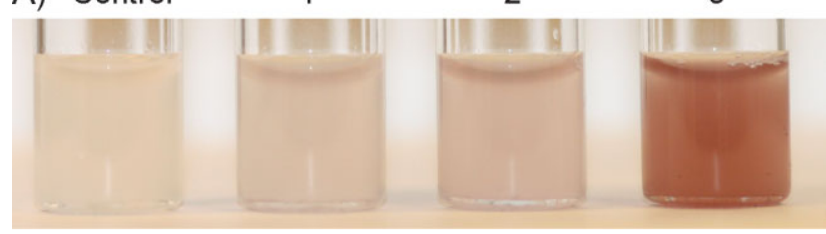
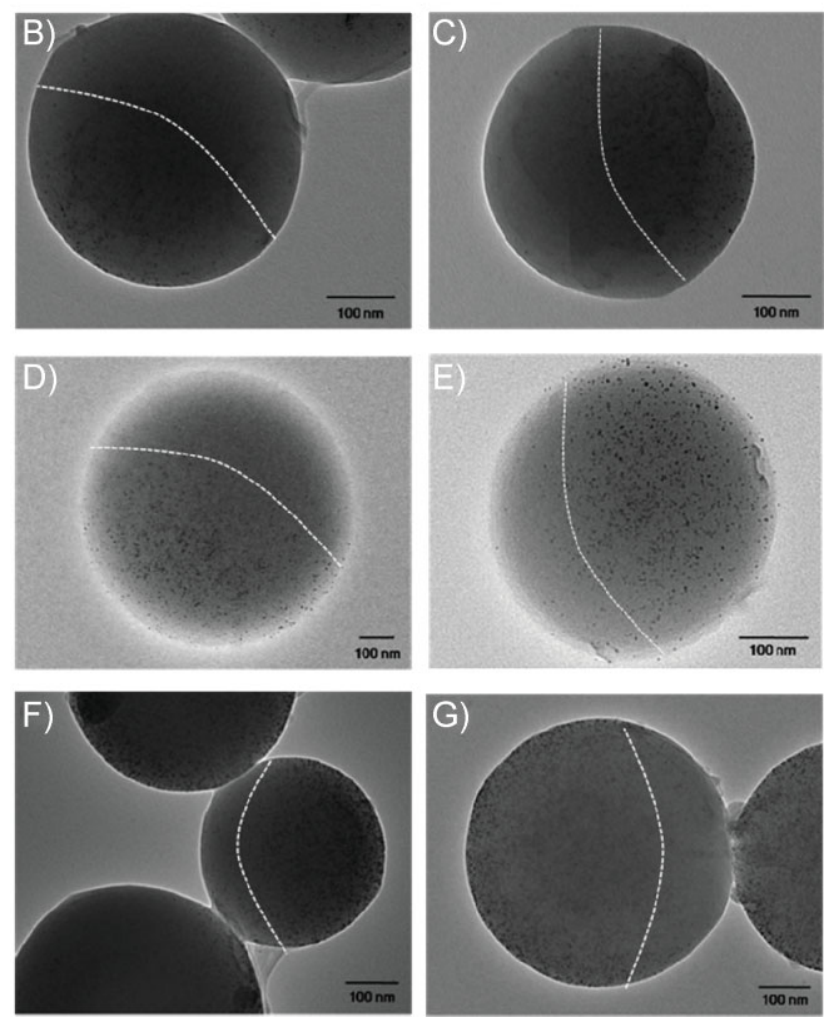

Figure 2. (A) Photographic images of aqueous suspensions of bicompartmental particles having gold NCs at different densities within one compartment (Control: ow/v \%, 1:0.18 w/v \%, $2: 0.36 \mathrm{w} / \mathrm{v} \%$, and $3: 0.72 \mathrm{w} / \mathrm{v} \%$ for the NC concentration in the jetting solution). (B)-(G) TEM images of the bicompartmental particles shown in (A), $(B, C)$ two separate TEM images of $(1),(D, E)$ TEM images of (2), and (F,G) TEM images of (3), respectively. Schematic dash lines are included in each image to guide the eye toward the boundary between two compartments. Scale bars are $100 \mathrm{~nm}$.

confined into one single compartment. In addition, the TEM images of the bicompartmental particles shown in Figure 2(B)-(G) confirm different densities of the gold NCs confined in the one compartment. In fact, the density of the gold NCs in one compartment of the particles was successively increased, as the concentration of the gold NCs in the corresponding jetting solutions increased [(B) \& (C) $0.18 \mathrm{w} / \mathrm{v} \%$; (D) \& (E) $0.36 \mathrm{w} / \mathrm{v} \%$; and (F) \& (G) $0.72 \mathrm{w} / \mathrm{v} \%$ for the concentration of the gold NCs in the jetting solutions, corresponding to (B) \& (C) 2.3 wt.-\%; (D) \& (E) 4.6 wt.-\%; and (F) \& (G) 8.8 wt.-\% for a theoretical weight ratio of the gold NCs in the compartment based on a dried state). These findings suggest that the density of the compartmentalized gold NCs in the anisotropic particles can be controlled by varying the concentration of the gold NCs in the jetting solution. Most importantly, analysis of the TEM images clearly show that the gold NCs with an approximate diameter of $4.3 \mathrm{~nm}$ (Figure S1 of Supporting Information $)^{[35,36]}$ were evenly distributed within one compartment of the bicompartmental particles. The diameter of the bicompartmental particles was $380-820 \mathrm{~nm}$. No severe aggregation of gold NCs was detected, based on TEM analysis, potentially due to a charge repulsion of sulfonate groups between the gold NCs in the process of the EHD cojetting. ${ }^{[35,36]}$ Furthermore, there was very little transfer of the gold NCs between compartments and out of the particles, implying that compartmentalization of the gold NCs was sustained in the course of the EHD co-jetting, thermal cross-linking, ultrasonication, and the subsequent swelling-drying procedures.

Figure 3 summarizes both qualitative and quantitative results obtained by CLSM and flow cytometry. The data indicate a high degree of anisotropy and a high fraction of the bicompartmental particles containing the gold NCs in the FITC compartment. As represented in Figure 3(A), a DIC image of the particles $(0.36 \mathrm{w} / \mathrm{v} \%$ for the gold NC concentration in the jetting solution) suspended in PBS at $\mathrm{pH} 7.4$ shows that the polymer particles have spherical shapes with relatively larger diameters $(2-4 \mu \mathrm{m})$, as compared to the diameters determined by TEM, which were substantially smaller than $1 \mu \mathrm{m}$, as shown in Figure 2 . Taken together, the data suggest that the thermal imidization reaction occurred homogeneously throughout the compartments of the polymer particles in spite of the presence of gold NCs (Figure S2 of Supporting Information) ${ }^{[11,14]}$ Moreover, the particles undergo isotropic swelling. Figure 3(B) is a CLSM image of green fluorescence emission taken for the same particle population of the DIC image in Figure 3(A). Figure 3 (C)-(E) are CLSM images of green fluorescence emission (FITC), red fluorescence emission (rhodamine B), and their fluorescence overlays of the bicompartmental polymer particles, respectively. The CLSM images indicate a high degree of anisotropy, even in the swollen state of the particles. The sharp interface between two compartments was maintained, although the concentration of the gold NCs in the FITC jetting solution increased from 0.18 to $0.72 \mathrm{w} / \mathrm{v} \%$ (Figure S3 of Supporting Information). Additionally, flow cytometry measurements were carried out to quantify the fraction of the bicompartmental particles based on the entire particle population. The data are summarized in Figure $3(\mathrm{~F})$ and $(\mathrm{G})$. Two batches of bicompartmental particles were separately prepared for this analysis: the bicompartmental particles having the gold NCs at the highest density within one compartment $(0.72 \mathrm{w} / \mathrm{v} \%$ for the gold NC concentration in one jetting solution) without any fluorescence dyes [Figure 3(F)], and with FITC-dextran (the compartment containing the gold 

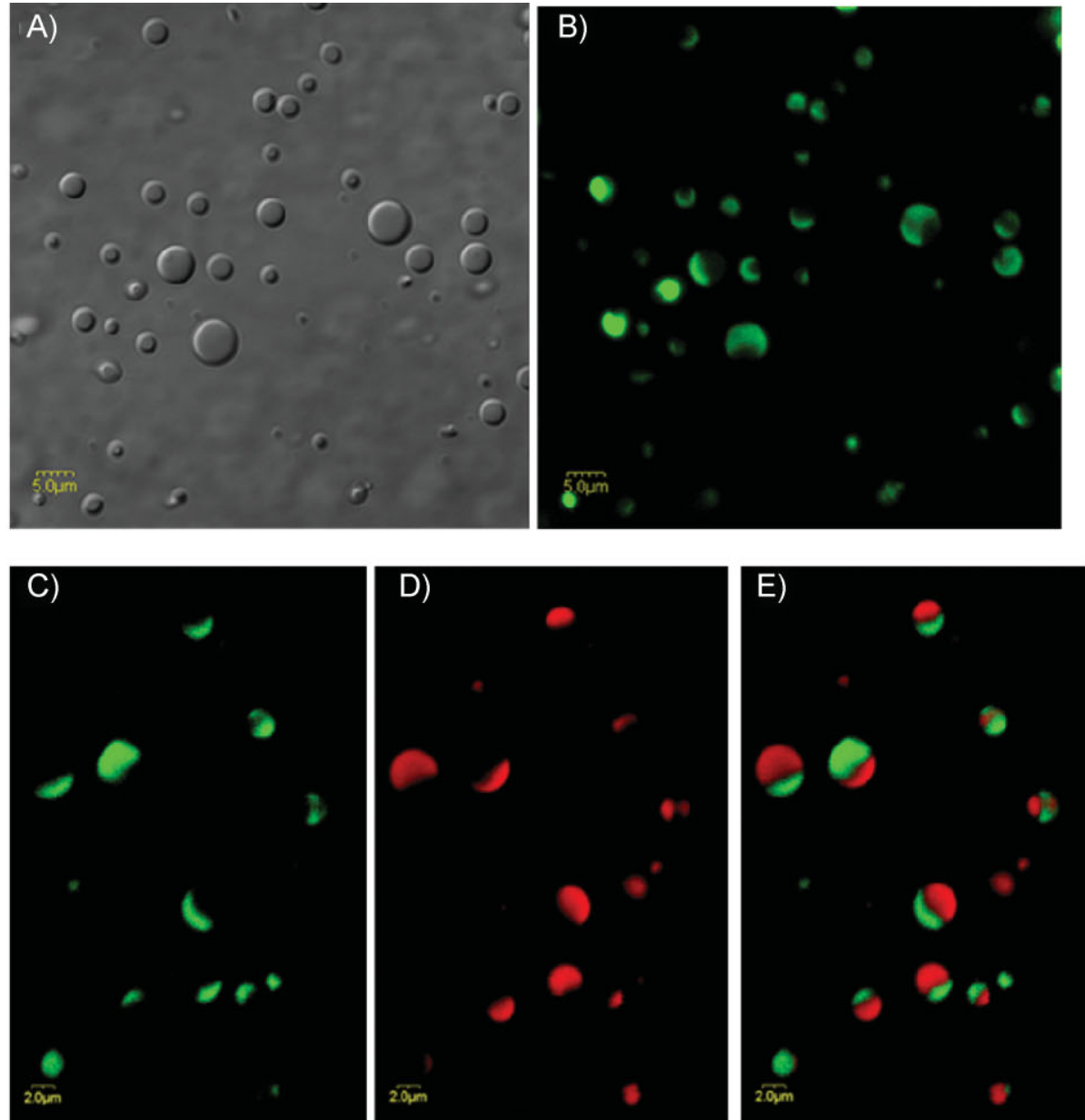

F)

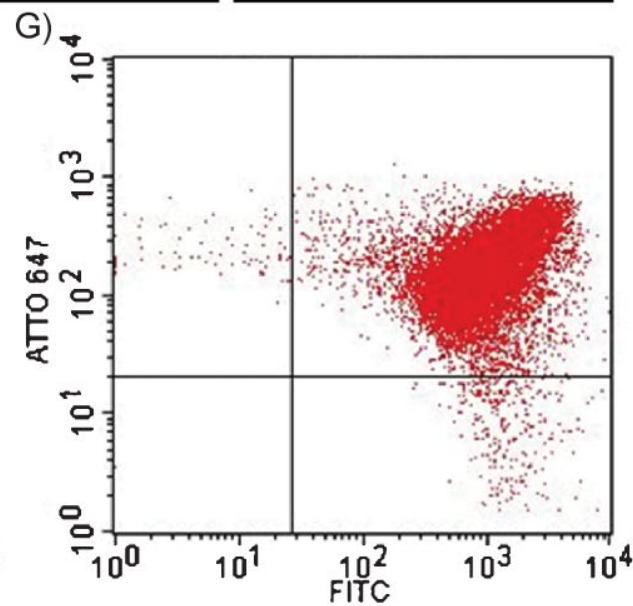

Figure 3. (A) DIC image and (B) CLSM image of FITC green fluorescence emission of bicompartmental polymer particles having gold NCs within one compartment ( $0.36 \mathrm{w} / \mathrm{v} \%$ for the NC concentration in the one jetting solution) when they were suspended in PBS at pH 7.4. (C)-(E) Their CLSM images of green fluorescence emission (FITC) in (C), red fluorescence emission (rhodamine B) in (D), and the overlays of green and red fluorescence emission in (E). (F, G) Flow cytometry density plots of bicompartmental particles having gold NCs at the highest density within one compartment ( $0.72 \mathrm{w} / \mathrm{v} \%$ for the NC concentration in the one jetting solution) without any fluorescence dyes (F) and with FITC-dextran and Atto 647-dextran at respective compartments (G). The image sets for $(A, B)$ and $(C-E)$ were taken in situ separately. The DIC image in (A) shows isotropically swollen particles in a spherical shape while the CLSM image in (B) represents that the particles have distinct green fluorescent compartments having the gold NCs. Scale bars are $5 \mu \mathrm{m}$ in $(A, B)$ and $2 \mu \mathrm{m}$ in $(C-E)$. 
NCs) and Atto 647-dextran [the counter compartment, Figure $3(\mathrm{G})$ ], respectively. The density plot of the bicompartmental particles having no fluorescence dyes in Figure 3(F) was included as a control. The use of this control group resulted in a baseline signal that was established by including over $99 \%$ for the particle population (data points in the lower left quadrant). The predetermined baseline signal was subtracted from the overall signal, when bicompartmental particles containing fluorescence dyes was measured, as indicated by a solid line of each axis in the density plots $[x$ - and $y$-axes in Figure $3(F)$ and $(G)]$. Figure $3(\mathrm{G})$ confirms that the fraction of bicompartmental particles with both FITC-dextran and Atto 647-dextran (the upper right quadrant) is higher than $98 \%$ of the total particle population, whereas the population of particles containing only one fluorescence polymer, FITC-dextran (the lower right quadrant) or Atto 647-dextran (the upper left quadrant) is 1.6 or $0.4 \%$, respectively.

Finally, SEM images are shown at different magnifications in Figure 4(A) and (B) to assess the surface morphology
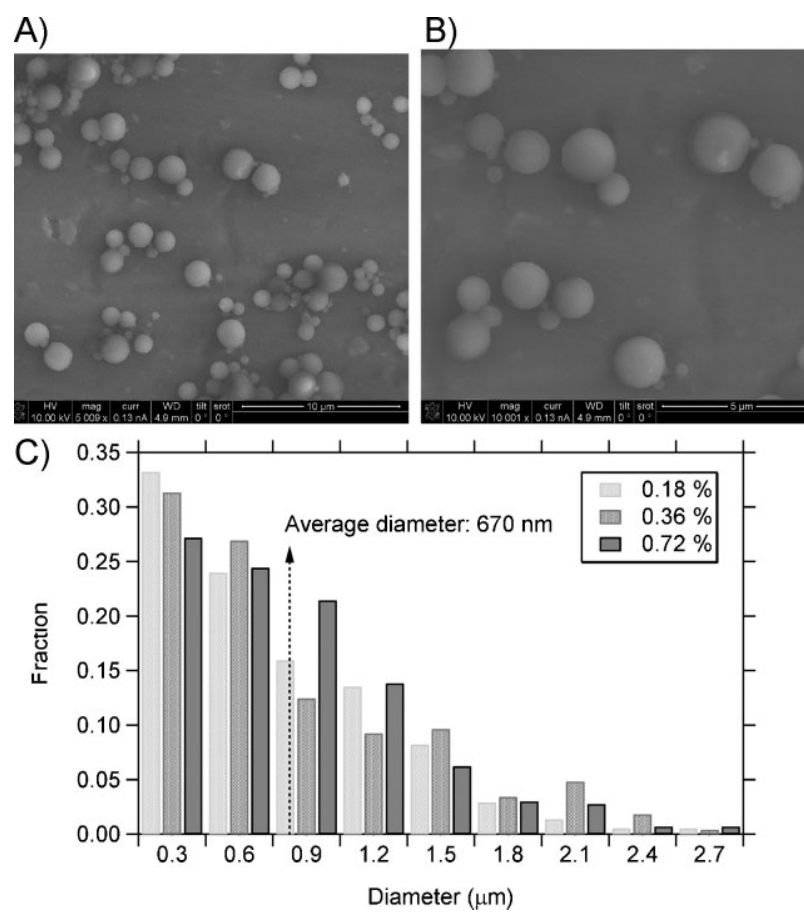

Figure 4. $(\mathrm{A}, \mathrm{B}) \mathrm{SEM}$ images and $(\mathrm{C})$ an overall average diameter and size distribution of bicompartmental polymer particles having gold NCs at different densities within one compartment (o.18, 0.36 , and $0.72 \mathrm{w} / \mathrm{v} \%$ for the NC concentration in the one jetting solution). (A,B) SEM images of the bicompartmental polymer particles with the gold NCs confined in the one compartment ( $0.36 \mathrm{w} / \mathrm{v} \%$ for the gold NC concentration in the jetting solution) at different magnifications. The particles with diameters above $1 \mu \mathrm{m}$ were selected for better SEM images. The average diameter and size distribution of the bicompartmental polymer particles were determined by measuring approximately $400-500$ particles in the SEM images. Scale bars are $10 \mu \mathrm{m}$ in (A) and $5 \mu \mathrm{m}$ in (B). of bicompartmental polymer particles $(0.36 \mathrm{w} / \mathrm{v} \%$ for the gold NC concentration in the jetting solution). The SEM images suggest that the particles are in a spherical form with an average diameter of $720 \mathrm{~nm}$. No significant amounts of beads-on-a-string morphologies were observed. These results confirm that the EHD co-jetting condition was efficiently controlled to minimize the beads-on-a-string morphology by optimizing the polymer concentration. Interparticle cross-linking during thermal curing was ruled out as well. Figure $4(C)$ shows size distributions of the bicompartmental polymer particles acquired from the SEM data sets. Three batches of the bicompartmental polymer particles with different densities of the gold NCs $(0.18,0.36$, and $0.72 \mathrm{w} / \mathrm{v} \%$ for the gold NC concentration in the jetting solution) were studied, and they have similar average diameters and size distributions, regardless of the different concentration of the gold $\mathrm{NCs}$ in the jetting solution $(0.18 \mathrm{w} / \mathrm{v} \%: 650 \pm 460 \mathrm{~nm}, 0.36 \mathrm{w} / \mathrm{v} \%: 720 \pm 550 \mathrm{~nm}$, $0.72 \mathrm{w} / \mathrm{v} \%: 700 \pm 470 \mathrm{~nm}$, as shown in an average diameter \pm S.D.), suggesting that the presence of the gold NCs in the jetting solution had no significant effect on the jet break-up during the EHD co-jetting.

\section{Conclusion}

Controlled compartmentalization of gold NCs within anisotropic polymer particles at the micro- and nanoscale was achieved by optimizing polymeric concentration of jetting solutions used for EHD co-jetting. The density of gold NCs in the bicompartmental polymer particles was controlled by simply varying the concentrations of gold NCs in the jetting solutions, which results in bicompartmental particles with different optical properties. Transfer of gold NCs across the compartment interface was negligible, and no substantial aggregation of the gold NCs was observed, even for higher densities of gold NCs. In addition, analysis of the CLSM images showed distinctive fluorescence boundaries in the anisotropic particles as well as isotropic swelling behavior. Furthermore, flow cytometry measurements revealed the fraction of bicompartmental particles to be more than $98 \%$ of the total particle population. The data strongly support that the preparative procedures employed in this research are reasonably well-established to compartmentalize gold NCs in anisotropic polymer particles at the micro- and nanoscale. Consequently, this study opens the possibility for synthesis of bicompartmental polymer particles with various functional nanomaterials, such as inorganic materials, metals, quantum dots, and carbon nanotubes within each compartment, which may be applied to multimodal cell targeting, medical imaging, and dynamic switching by external stimuli. 
Acknowledgements: The authors specially thank Srijanani Bhaskar, Kyung-Ho Roh, Mutsumi Yoshida, Aftin Ross, and Nancy Tseng in the Department of Chemical Engineering at the University of Michigan for their valuable help and discussion. This project was partly supported by a grant from the American Cancer Society (RSG-08-284-01-CDD) and Seventh Sense Biosystems.

Received: August 18, 2009; Revised: September 22, 2009; Published online: December 1, 2009; DOI: 10.1002/ marc.200900597

Keywords: anisotropy; composites; fluorescence; Janus particle; nanocrystals; polymer

[1] S. Mitragotri, J. Lahann, Nat. Mater. 2009, 8, 15.

[2] A. Sun, J. Lahann, Soft Matter 2009, 5, 1555.

[3] M. Yoshida, J. Lahann, ACS Nano 2008, 2, 1101.

[4] J. R. Howse, R. A. L. Jones, A. J. Ryan, T. Gough, R. Vafabakhsh, R. Golestanian, Phys. Rev. Lett. 2007, 99, 048102.

[5] S. Gangwal, O. J. Cayre, O. D. Velev, Langmuir 2008, 24, 13312.

[6] A. Ghosh, N. K. Sheridon, P. Fischer, Small 2008, 4, 1956.

[7] A. Goyal, C. K. Hall, O. D. Velev, Phys. Rev. E 2008, 77, 0314401.

[8] J. R. Millman, K. H. Bhatt, B. G. Prevo, O. D. Velev, Nat. Mater. 2005, 4, 98.

[9] S. K. Smoukov, S. Gangwal, M. Marquez, O. D. Velev, Soft Matter 2009, 5, 1285.

[10] S. Bhaskar, K. H. Roh, X. W. Jiang, G. L. Baker, J. Lahann, Macromol. Rapid Commun. 2008, 29, 1655.

[11] K. H. Roh, D. C. Martin, J. Lahann, Nat. Mater. 2005, 4, 759.

[12] K. H. Roh, D. C. Martin, J. Lahann, J. Am. Chem. Soc. 2006, 128, 6796.

[13] K. H. Roh, M. Yoshida, J. Lahann, Langmuir 2007, 23, 5683.

[14] M. Yoshida, K. H. Roh, J. Lahann, Biomaterials 2007, 28, 2446.

[15] T. Nisisako, T. Torii, Adv. Mater. 2007, 19, 1489.

[16] T. Nisisako, T. Torii, Lab on a Chip 2008, 8, 287.

[17] T. Nisisako, T. Torii, T. Takahashi, Y. Takizawa, Adv. Mater. 2006, 18, 1152.

[18] D. Suzuki, H. Kawaguchi, Colloid Polym. Sci. 2006, 284, 1471.
[19] D. Suzuki, S. Tsuji, H. Kawaguchi, J. Am. Chem. Soc. 2007, 129, 8088.

[20] D. K. Hwang, J. Oakey, M. Toner, J. A. Arthur, K. S. Anseth, S. Lee, A. Zeiger, K. J. van Vliet, P. S. Doyle, J. Am. Chem. Soc. 2009, 131, 4499.

[21] P. Panda, K. P. Yuet, T. A. Hatton, P. S. Doyle, Langmuir 2009, 25, 5986.

[22] A. S. Utada, E. Lorenceau, D. R. Link, P. D. Kaplan, H. A. Stone, D. A. Weitz, Science 2005, 308, 537.

[23] S. O. Xu, Z. H. Nie, M. Seo, P. Lewis, E. Kumacheva, H. A. Stone, P. Garstecki, D. B. Weibel, I. Gitlin, G. M. Whitesides, Angew. Chem., Int. Ed. 2005, 44, 724.

[24] A. Walther, M. Drechsler, S. Rosenfeldt, L. Harnau, M. Ballauff, V. Abetz, A. H. E. Muller, J. Am. Chem. Soc. 2009, 131, 4720.

[25] A. Walther, A. H. E. Muller, Soft Matter 2008, 4, 663.

[26] A. Walther, J. Yuan, V. Abetz, A. H. E. Muller, Nano Lett. 2009, 9 , 2026.

[27] Z. H. Nie, W. Li, M. Seo, S. Q. Xu, E. Kumacheva, J. Am. Chem. Soc. 2006, 128, 9408.

[28] S. Bhaskar, J. Lahann, J. Am. Chem. Soc. 2009, 131, 6650.

[29] Z. Y. Liu, D. D. L. Sun, P. Guo, J. O. Leckie, Nano Lett. 2007, 7, 1081.

[30] A. Jaworek, A. T. Sobczyk, J. Electrostatics 2008, 66, 197.

[31] A. Kazemi, J. Lahann, Small 2008, 4, 1756.

[32] S. Bhaskar, J. Hitt, S. W. L. Chang, J. Lahann, Angew. Chem., Int. Ed. 2009, 48, 4589.

[33] H. Nandivada, X. W. Jiang, J. Lahann, Adv. Mater. 2007, 19, 2197.

[34] K. Nakata, Y. Hu, O. Uzun, O. Bakr, F. Stellacci, Adv. Mater. 2008, 20, 4294.

[35] O. Uzun, Y. Hu, A. Verma, S. Chen, A. Centrone, F. Stellacci, Chem. Commun. 2008, 196.

[36] A. Verma, O. Uzun, Y. H. Hu, Y. Hu, H. S. Han, N. Watson, S. L. Chen, D. J. Irvine, F. Stellacci, Nat. Mater. 2008, 7, 588.

[37] A. M. Jackson, J. W. Myerson, F. Stellacci, Nat. Mater. 2004, 3, 330.

[38] A. Barrero, A. M. Ganan-Calvo, J. Davila, A. Palacio, E. Gomez-Gonzalez, Phys. Rev. E 1998, 58, 7309.

[39] A. Barrero, A. M. Ganan-Calvo, J. Davila, A. Palacios, E. Gomez-Gonzalez, J. Electrostatics 1999, 47, 13.

[40] A. Barrero, I. G. Loscertales, Ann. Rev. Fluid Mech. 2007, 39, 89. 